\title{
Pseudospin-electron model spectrum in alloy analogy approximation
}

\author{
I.V.Stasyuk, V.O.Krasnov \\ Institute for Condensed Matter Physics of the National Academy of Sciences of Ukraine, \\ 1 Svientsitskii Str., 79011 Lviv, Ukraine
}

Received January 26, 2006

Energy spectrum of the pseudospin-electron model is investigated in the alloy analogy approximation within the framework of the dynamical mean field theory (DMFT). The case of two near Hubbard-like electron subbands, which determine the location of chemical potential at a certain set of model parameter values, is considered. The conditions of gap appearance in the spectrum are established. The effect of the asymmetry field $h$ and tunneling-like splitting of levels in the local anharmonic well on the critical value $U_{\text {crit }}$ of the on-site Hubbard interaction constant is investigated.

Key words: dynamical mean-filed theory, irreducible Green's functions, pseudospin-electron model, alloy analogy approximation

PACS: $71.10 . F d, 71.38 .+i$

\section{Introduction}

Pseudospin-electron model (PEM) is one of the models that are used in the physics of stronglycorrelated electron systems in recent years. The application of the model to high-temperature superconductors allows us, for example, to describe the thermodynamics of anharmonic oxygen ion subsystem and explain the occurrence of inhomogeneous states and the bistability phenomena (see in [1]). In this model we take into consideration the dynamics of locally anharmonic structural elements (using pseudospin variables to describe them), the interaction between pseudospins and electrons and the asymmetry of local anharmonic potential wells. The electron subsystem is described by the Hubbard Hamiltonian.

It is simple to solve PEM in the case of zero electron transfer or without Coulomb interaction. When $W \gg U$ and $U \gg W$ (where $U$ is the Coulomb potential of single-site electron interaction, and $W$ is the bandwidth) the consideration can be based on the perturbation theory. If $U \sim W$, the perturbation approach cannot be used, and dynamical mean-field theory (DMFT) [2-4] is the most successful. In DMFT method the Hamiltonian with strong correlations is taken in the infinite space dimension $(d \rightarrow \infty)$ limit; this leads to reformulation of the problem and transition to the solution of the single-site problem described by effective Hamiltonian. Only for the simplest cases such as mobile particles in the Falicov-Kimball model one can solve this problem analytically. There are also some approximate analytical approaches, such as: Hubbard-I, Hubbard-III, alloy analogy (AA), modified alloy analogy (MAA) etc., see [5].

The alloy analogy approximation for the single-site problem is used for pseudo-spin-electron model in this article. Our task is to study electron spectrum and find the conditions of the gap appearance. The previous consideration of this problem in the Hubbard-I approximation [6] revealed complicated structure of the spectrum and the presence of a certain number of subbands (their appearance is caused by the correlational splitting due to Coulomb correlation $U$ and interaction $g$ of electrons with pseudospins). Contrary to this approach, when subbands are always energetically separated at the arbitrary $U$ and $g$ values, an AA approximation allows us to describe transitions with the joining of some subband and the disappearance of the gap. We consider this question 
based on two subbands which are close to each other at certain values of the asymmetry field $h$, the chemical potential of electrons being placed in the region of these subbands.

\section{Hamiltonian of the model}

The Hamiltonian of pseudospin-electron model is:

$$
H=\sum_{i} H_{i}+\sum_{\langle i, j\rangle, \sigma} t_{i j} a_{i \sigma}^{+} a_{j \sigma}
$$

where in the single-site part of the Hamiltonian $U$ is Coulomb repulsion, $\Omega$ is the tunneling-like level splitting, $g$ is the pseudospin-electron interaction and $h$ is asymmetry of the local anharmonic potential:

$$
H_{i}=U n_{i, \uparrow} n_{i, \downarrow}+E_{0}\left(n_{i, \uparrow}+n_{i, \downarrow}\right)+g\left(n_{i, \uparrow}+n_{i, \downarrow}\right) S_{i}^{z}-\Omega S_{i}^{x}-h S_{i}^{z} .
$$

The second term in (1) describes the electron site-to-site hopping.

The single-site Hamiltonian is considered to be the zero-order with respect to the electron transfer. It is useful to introduce the following standard single-site basis $|R\rangle=\left|n_{i, \uparrow}, n_{i, \downarrow}, S_{i}^{z}\right\rangle$, with eight eigenvectors [7]:

$$
\begin{array}{ll}
|1\rangle=\left|0,0, \frac{1}{2}\right\rangle, \quad|2\rangle=\left|1,1, \frac{1}{2}\right\rangle, \quad|3\rangle=\left|0,1, \frac{1}{2}\right\rangle, \quad|4\rangle=\left|1,0, \frac{1}{2}\right\rangle, \\
|\widetilde{1}\rangle=\left|0,0,-\frac{1}{2}\right\rangle, \quad|\widetilde{2}\rangle=\left|1,1,-\frac{1}{2}\right\rangle, \quad|\widetilde{3}\rangle=\left|0,1,-\frac{1}{2}\right\rangle, \quad|\widetilde{4}\rangle=\left|1,0,-\frac{1}{2}\right\rangle .
\end{array}
$$

Using Hubbard X-operators, that act in the space of such eigenvectors, we can write down the electron annihilation (creation) operators and pseudospin operators from the initial Hamiltonian as follows:

$$
\begin{array}{ll}
a_{i, \uparrow}=\mathrm{X}_{i}^{14}+\mathrm{X}_{i}^{32}+\mathrm{X}_{i}^{\widetilde{14}}+\mathrm{X}_{i}^{\widetilde{3}}, & a_{i, \downarrow}=\mathrm{X}_{i}^{13}-\mathrm{X}_{i}^{42}+\mathrm{X}_{i}^{\widetilde{13}}-\mathrm{X}_{i}^{\widetilde{42}}, \\
a_{i, \downarrow}^{+}=\mathrm{X}_{i}^{31}-\mathrm{X}_{i}^{24}+\mathrm{X}_{i}^{\widetilde{31}}-\mathrm{X}_{i}^{\widetilde{24}}, \quad a_{i, \uparrow}^{+}=\mathrm{X}_{i}^{41}+\mathrm{X}_{i}^{23}+\mathrm{X}_{i}^{\widetilde{41}}+\mathrm{X}_{i}^{\widetilde{23}} \\
n_{i, \uparrow}=\mathrm{X}_{i}^{44}+\mathrm{X}_{i}^{22}+\mathrm{X}_{i}^{\widetilde{44}}+\mathrm{X}_{i}^{\widetilde{2} \widetilde{2}}, \quad n_{i, \downarrow}=\mathrm{X}_{i}^{33}+\mathrm{X}_{i}^{22}+\mathrm{X}_{i}^{\widetilde{33}}+\mathrm{X}_{i}^{\widetilde{22}} \\
S_{i}^{z}=\frac{1}{2}\left(\mathrm{X}_{i}^{11}-\mathrm{X}_{i}^{\widetilde{1}}+\mathrm{X}_{i}^{22}-\mathrm{X}_{i}^{\widetilde{2}}+\mathrm{X}_{i}^{33}-\mathrm{X}_{i}^{\widetilde{3}}+\mathrm{X}_{i}^{44}-\mathrm{X}_{i}^{\widetilde{4}}\right) \\
S_{i}^{x}=\frac{1}{2}\left(\mathrm{X}_{i}^{1 \widetilde{1}}+\mathrm{X}_{i}^{\widetilde{1} 1}+\mathrm{X}_{i}^{2 \widetilde{2}}+\mathrm{X}_{i}^{\widetilde{2} 2}+\mathrm{X}_{i}^{3 \widetilde{3}}+\mathrm{X}_{i}^{\widetilde{3} 3}+\mathrm{X}_{i}^{\widetilde{4} 4}+\mathrm{X}_{i}^{4 \widetilde{4}}\right) .
\end{array}
$$

The Hamiltonian $H_{i}$ can be expressed by means of X-operators as follows:

$$
\begin{aligned}
H_{i}= & \left(E_{0}+\frac{g}{2}-\frac{h}{2}\right)\left(\mathrm{X}_{i}^{33}+\mathrm{X}_{i}^{44}\right)+\left(E_{0}-\frac{g}{2}+\frac{h}{2}\right)\left(\mathrm{X}_{i}^{\widetilde{33}}+\mathrm{X}_{i}^{\widetilde{4}}\right)+\frac{h}{2}\left(\mathrm{X}_{i}^{\widetilde{1} 1}-\mathrm{X}_{i}^{11}\right) \\
& +\left(U+2 E_{0}+g-\frac{h}{2}\right) \mathrm{X}_{i}^{22}+\left(U+2 E_{0}-g+\frac{h}{2}\right) \mathrm{X}_{i}^{\widetilde{2} \widetilde{2}} \\
& -\frac{\Omega}{2}\left(\mathrm{X}_{i}^{\widetilde{1}}+\mathrm{X}_{i}^{\widetilde{1} 1}+\mathrm{X}_{i}^{2 \widetilde{2}}+\mathrm{X}_{i}^{\widetilde{2} 2}+\mathrm{X}_{i}^{3 \widetilde{3}}+\mathrm{X}_{i}^{\widetilde{3} 3}+\mathrm{X}_{i}^{\widetilde{4} 4}+\mathrm{X}_{i}^{\widetilde{4}}\right) .
\end{aligned}
$$

This Hamiltonian is diagonal in the case $\Omega=0$, but if the tunneling splitting is non-zero we have to use transformation [6]

$$
\left(\begin{array}{c}
R \\
\widetilde{R}
\end{array}\right)=\left(\begin{array}{cc}
\cos \phi_{r} & \sin \phi_{r} \\
-\sin \phi_{r} & \cos \phi_{r}
\end{array}\right)\left(\begin{array}{l}
r \\
\widetilde{r}
\end{array}\right)
$$

to diagonalize it.

Here

$$
\cos \left(2 \phi_{r}\right)=\frac{n_{r} g-h}{\sqrt{\left(n_{r} g-h\right)^{2}+\Omega^{2}}}, \quad n_{1}=0, \quad n_{2}=2, \quad n_{3}=n_{4}=1
$$


In this way we have

$$
\begin{gathered}
H=\sum_{i, r} \varepsilon_{r} X_{i}^{r r}+\sum_{\langle i, j\rangle, \sigma} t_{i j} a_{i \sigma}^{+} a_{j \sigma}, \\
\varepsilon_{1, \tilde{1}}= \pm \frac{1}{2} \sqrt{h^{2}+\Omega^{2}}, \quad \varepsilon_{2, \widetilde{2}}=2 E_{0}+U \pm \frac{1}{2} \sqrt{(2 g-h)^{2}+\Omega^{2}} \\
\varepsilon_{3, \widetilde{3}}=\varepsilon_{4, \widetilde{4}}=E_{0} \pm \frac{1}{2} \sqrt{(g-h)^{2}+\Omega^{2}} .
\end{gathered}
$$

After transformation (6) the operators of the electron creation and annihilation can be written using the $\mathrm{X}$-operators as follows:

$$
a_{i, \sigma}=\sum_{m, n} A_{m n}^{\sigma} X_{i}^{n m}, \quad a_{i, \sigma}^{+}=\sum_{m, n} A_{m n}^{\sigma} X_{i}^{m n}
$$

where $X_{i}^{m n}=|m\rangle\langle n|$ and:

$$
\begin{array}{ll}
A_{41}^{\uparrow}=A_{\widetilde{11}}^{\uparrow}=\cos \left(\phi_{4}-\phi_{1}\right), & A_{23}^{\uparrow}=A_{\widetilde{2} 3}^{\uparrow}=\cos \left(\phi_{2}-\phi_{3}\right), \\
A_{\widetilde{4} 1}^{\uparrow}=-A_{4 \widetilde{1}}^{\uparrow}=\sin \left(\phi_{4}-\phi_{1}\right), & A_{\tilde{2} 3}^{\uparrow}=-A_{2 \widetilde{3}}^{\uparrow}=\sin \left(\phi_{2}-\phi_{3}\right), \\
A_{31}^{\downarrow}=A_{\widetilde{31}}^{\downarrow}=\cos \left(\phi_{3}-\phi_{1}\right), & A_{24}^{\downarrow}=A_{\widetilde{2} 4}^{\downarrow}=-\cos \left(\phi_{2}-\phi_{4}\right), \\
A_{\tilde{3} 1}^{\downarrow}=-A_{3 \widetilde{1}}^{\downarrow}=\sin \left(\phi_{3}-\phi_{1}\right), & A_{\tilde{2} 4}^{\downarrow}=-A_{2 \widetilde{4}}^{\downarrow}=-\sin \left(\phi_{2}-\phi_{4}\right) .
\end{array}
$$

\section{Dynamical mean field theory}

The transition to the $d=\infty$ in the DMFT approach is accompanied by scaling the electron transfer parameter:

$$
t=\frac{t^{*}}{\sqrt{d}} .
$$

In particular, the self-energy part of the electron Green's function becomes purely local [3,4]:

$$
\Sigma_{i j, \sigma}(\omega)=\Sigma_{\sigma} \delta_{i j}, \quad d=\infty .
$$

The Fourier-transform $\Sigma_{i j, \sigma}(\omega)$ is hence momentum-independent:

$$
\Sigma_{\sigma}(\vec{k}, \omega)=\Sigma_{\sigma}(\omega)
$$

The electron Green's function in $(k, \omega)$ representation

$$
G_{k}^{\sigma}(\omega)=\sum_{i-j} \mathrm{e}^{\mathrm{i} \vec{k}\left(\vec{R}_{i}-\vec{R}_{j}\right)} G_{i j, \sigma}(\omega)
$$

can be expressed as:

$$
G_{k}^{\sigma}(\omega)=\frac{1}{\left[\Xi_{\sigma}(\omega)\right]^{-1}-t_{k}},
$$

where $\Xi_{\sigma}(\omega)$ is the irreducible part (in the diagrammatic representation) according to Larkin. To calculate the $\Xi_{\sigma}(\omega)$ function the effective single-site problem is used. The transition to this problem corresponds to the replacement [8]:

$$
\mathrm{e}^{-\beta H} \rightarrow \mathrm{e}^{-\beta H_{\text {eff }}}=\mathrm{e}^{-\beta H_{0}} \times T \exp \left\{-\int_{0}^{\beta} \mathrm{d} \tau \int_{0}^{\beta} \mathrm{d} \tau^{\prime} \sum_{\sigma} J_{\sigma}\left(\tau-\tau^{\prime}\right) a_{\sigma}^{+}(\tau) a_{\sigma}\left(\tau^{\prime}\right)\right\},
$$


where

$$
H_{0}=H_{i}
$$

and $J_{\sigma}\left(\tau-\tau^{\prime}\right)$ - is an effective time-dependant field (coherent potential) which is self-consistently determined from the condition that the same self-energy part $\Xi_{\sigma}(\omega)$ determines the lattice function $G_{k}^{\sigma}(\omega)$ as well as the Green's function $G_{\sigma}^{(a)}(\omega)$ of the effective single-site problem:

$$
G_{\sigma}^{(a)}(\omega)=\frac{1}{\left[\Xi_{\sigma}(\omega)\right]^{-1}-J_{\sigma}(\omega)} .
$$

In this case:

$$
G_{\sigma}^{(a)}=G_{i i, \sigma}(\omega)=\frac{1}{N} \sum_{k} G_{k}^{\sigma}(\omega) .
$$

The set of simultaneous equations (15), (18), (19) becomes closed when it is supplemented with the functional dependence

$$
G_{\sigma}^{(a)}(\omega)=f\left(\left[J_{\sigma}(\omega)\right]\right)
$$

which is obtained as the result of solving the effective single-site problem with the statistical operator $\exp \left(-\beta H_{\text {eff }}\right)$.

\section{The equations of motion for the Green's functions}

Let us reformulate a single-site problem explicitly introducing an effective Hamiltonian

$$
\widetilde{H}_{\text {eff }}=H_{0}+V \sum_{\sigma}\left(a_{\sigma}^{+} \xi_{\sigma}+\xi_{\sigma}^{+} a_{\sigma}\right)+H_{\xi},
$$

$H_{0}$ is the single-site part of the Hamiltonian (8) in the X-operator representation. Here the electron hopping from a given site to the effective medium is taken into account. The Hamiltonian of the effective medium $H_{\xi}$ is unknown, but the Green's function

$$
\Upsilon_{\sigma}(\omega)=\left\langle\left\langle\xi_{\sigma} \mid \xi_{\sigma}^{+}\right\rangle\right\rangle_{\omega}^{\left(H_{\xi}\right)}
$$

for auxiliary fermions is considered as the given function which must be determined self-consistently. It was shown [9] that

$$
\left\langle\exp \left(-\beta \widetilde{H}_{\mathrm{eff}}\right)\right\rangle^{\left(H_{\xi}\right)}=\exp \left(-\beta H_{\mathrm{eff}}\right)
$$

and the relation

$$
2 \pi V^{2} \Upsilon_{\sigma}(\omega)=J_{\sigma}(\omega)
$$

takes place in this case.

In our case we have the following Hamiltonian which we use in order to find the Green's function $G_{\sigma}^{(a)}(\omega)$ and obtain an explicit form of functional relation (20)

$$
\widetilde{H}_{\text {eff }}=\sum_{r} \varepsilon_{r} X^{r r}+V\left(a_{\uparrow}^{+} \xi_{\uparrow}+\xi_{\uparrow}^{+} a_{\uparrow}+a_{\downarrow}^{+} \xi_{\downarrow}+\xi_{\downarrow}^{+} a_{\downarrow}\right)+H_{\xi} .
$$

The problem is described in terms of auxiliary Fermi-field $\xi, \xi^{+}$; the operators $a_{\uparrow}, a_{\downarrow}$ are expressed in terms of the X-operators according to (10). In this case the required functions $G_{\sigma}^{(a)}(\omega)(\sigma=\uparrow, \downarrow)$ are

$$
\begin{aligned}
& G_{\uparrow}^{(a)}(\omega) \equiv\left\langle\left\langle a_{\uparrow} \mid a_{\uparrow}^{+}\right\rangle\right\rangle=\left\langle\left\langle\left(X^{14}+X^{\widetilde{14}}\right) \cos \left(\phi_{4}-\phi_{1}\right)+\left(X^{1 \widetilde{4}}-X^{\widetilde{1} 4}\right) \sin \left(\phi_{4}-\phi_{1}\right)\right.\right. \\
& \quad+\left(X^{32}+X^{\widetilde{32}}\right) \cos \left(\phi_{2}-\phi_{3}\right)+\left(X^{3 \widetilde{2}}-X^{\widetilde{32}}\right) \sin \left(\phi_{2}-\phi_{3}\right) \mid\left(X^{41}+X^{\widetilde{41}}\right) \cos \left(\phi_{4}-\phi_{1}\right) \\
& \left.\left.\quad+\left(X^{4 \widetilde{1}}-X^{\widetilde{4} 1}\right) \sin \left(\phi_{4}-\phi_{1}\right)+\left(X^{23}+X^{\widetilde{23}}\right) \cos \left(\phi_{2}-\phi_{3}\right)+\left(X^{2 \widetilde{3}}-X^{\widetilde{2} 3}\right) \sin \left(\phi_{2}-\phi_{3}\right)\right\rangle\right\rangle,
\end{aligned}
$$




$$
\begin{aligned}
G_{\downarrow}^{(a)}(\omega) \equiv\left\langle\left\langle a_{\downarrow} \mid a_{\downarrow}^{+}\right\rangle\right\rangle=\left\langle\left\langle\left(X^{13}+X^{\widetilde{13}}\right) \cos \left(\phi_{3}-\phi_{1}\right)+\left(X^{1 \widetilde{3}}-X^{\widetilde{1} 3}\right) \sin \left(\phi_{3}-\phi_{1}\right)\right.\right. \\
\quad-\left(X^{42}+X^{\widetilde{42}}\right) \cos \left(\phi_{2}-\phi_{4}\right)+\left(X^{\widetilde{4} 2}-X^{4 \widetilde{2}}\right) \sin \left(\phi_{2}-\phi_{4}\right) \mid\left(X^{31}+X^{\widetilde{3} \widetilde{1}}\right) \cos \left(\phi_{3}-\phi_{1}\right) \\
\left.\left.\quad+\left(X^{\widetilde{3} 1}-X^{3 \widetilde{1}}\right) \sin \left(\phi_{3}-\phi_{1}\right)-\left(X^{24}+X^{\widetilde{24}}\right) \cos \left(\phi_{2}-\phi_{4}\right)+\left(X^{2 \widetilde{4}}-X^{\widetilde{2} 4}\right) \sin \left(\phi_{2}-\phi_{4}\right)\right\rangle\right\rangle .
\end{aligned}
$$

We have to calculate the Green's functions $\left\langle\left\langle X^{m n} \mid X^{p q}\right\rangle\right\rangle_{\omega}$ to find $G_{\sigma}^{(a)}(\omega)$, and for this purpose we write the equations of motion for X-operators. For example, for $X^{14}$ operator we have

$$
\begin{aligned}
& \mathrm{i} \frac{\mathrm{d}}{\mathrm{d} t} X^{14}(t)=\left[X^{14}, \widetilde{H}_{\mathrm{eff}}\right]=\left(\varepsilon_{4}-\varepsilon_{1}\right) X^{14}+V \cos \left(\phi_{4}-\phi_{1}\right)\left(X^{11}+X^{44}\right) \xi_{\uparrow} \\
& \quad+V \sin \left(\phi_{4}-\phi_{1}\right)\left(X^{\widetilde{4} 4}+X^{1 \widetilde{1}}\right) \xi_{\uparrow}+V X^{12} \xi_{\downarrow}^{+} \cos \left(\phi_{2}-\phi_{4}\right)+V X^{1 \widetilde{2}} \xi_{\downarrow}^{+} \sin \left(\phi_{2}-\phi_{4}\right) \\
& \quad+V X^{34} \xi_{\downarrow} \cos \left(\phi_{3}-\phi_{1}\right)+V X^{\widetilde{3} 4} \xi_{\downarrow} \sin \left(\phi_{3}-\phi_{1}\right) .
\end{aligned}
$$

Similar commutators had to be written for all the other X-operators from $(26,27)$. As a result, we get, for example, the following equation for $\left\langle\left\langle X^{14} \mid X^{41}\right\rangle\right\rangle_{\omega}$ function using the relation (28)

$$
\begin{aligned}
(\omega- & \left.\varepsilon_{4}+\varepsilon_{1}\right)\left\langle\left\langle X^{14} \mid X^{41}\right\rangle\right\rangle=\frac{1}{2 \pi}\left\langle X^{11}+X^{44}\right\rangle+V\left\langle\left\langle\left(X^{11}+X^{44}\right) \cos \left(\phi_{4}-\phi_{1}\right) \xi_{\uparrow} \mid X^{41}\right\rangle\right\rangle \\
& +V\left\langle\left\langle\sin \left(\phi_{4}-\phi_{1}\right)\left(X^{\widetilde{4} 4}+X^{1 \widetilde{1}}\right) \xi_{\uparrow} \mid X^{41}\right\rangle\right\rangle+V\left\langle\left\langle X^{12} \xi_{\downarrow}^{+} \cos \left(\phi_{2}-\phi_{4}\right) \mid X^{41}\right\rangle\right\rangle \\
& +V\left\langle\left\langle X^{1 \widetilde{2}} \xi_{\downarrow}^{+} \sin \left(\phi_{2}-\phi_{4}\right) \mid X^{41}\right\rangle\right\rangle+V\left\langle\left\langle X^{34} \xi_{\downarrow} \cos \left(\phi_{3}-\phi_{1}\right) \mid X^{41}\right\rangle\right\rangle \\
& +V\left\langle\left\langle X^{\widetilde{3} 4} \xi_{\downarrow} \sin \left(\phi_{3}-\phi_{1}\right) \mid X^{41}\right\rangle\right\rangle .
\end{aligned}
$$

Let us separate in Green's functions of higher order the irreducible parts using the method developed in $[10,11]$. We express derivatives $\mathrm{i} \frac{\mathrm{d}}{\mathrm{d} t} X^{p q}(t)$ as sums of regular (projected on the subspace formed by the Fermi-type operators $X^{p q}$ ) and irregular parts. The latter ones describe an inelastic quasiparticle scattering. Now, one can write down the (28) commutator as:

$$
\begin{aligned}
{\left[X^{14}, \widetilde{H}_{\mathrm{eff}}\right]=} & \left(\varepsilon_{4}-\varepsilon_{1}\right) X^{14}+\alpha_{1}^{14} X^{14}+\alpha_{2}^{14} X^{\widetilde{14}}+\alpha_{3}^{14} X^{1 \widetilde{4}}+\alpha_{4}^{14} X^{\widetilde{14}}+\alpha_{5}^{14} X^{32} \\
& +\alpha_{6}^{14} X^{\widetilde{32}}+\alpha_{7}^{14} X^{\widetilde{32}}+\alpha_{8}^{14} X^{3 \widetilde{2}}+Z^{14}
\end{aligned}
$$

Operators $Z^{p q}$ are defined as orthogonal to operators from the basic subspace:

$$
\left\langle\left\{Z^{p q}, X^{r s}\right\}\right\rangle=0 .
$$

These equations determine the coefficients $\alpha_{i}^{m n}$.

For these latter we have the following set of equations:

$$
\left(\begin{array}{cccc}
\left\langle X^{11}+X^{44}\right\rangle & 0 & \left\langle X^{4 \overline{4}}\right\rangle & \left\langle X^{\widetilde{1} 1}\right\rangle \\
0 & \left\langle X^{\widetilde{11}}+X^{\widetilde{4}}\right\rangle & \left\langle X^{1 \overline{4}}\right\rangle & \left\langle X^{\widetilde{4} 4}\right\rangle \\
\left\langle X^{\tilde{4} 4}\right\rangle & \left\langle X^{\widetilde{1} 1}\right\rangle & \left\langle X^{11}+X^{\widetilde{4}}\right\rangle & 0 \\
\left\langle X^{1 \widetilde{1}}\right\rangle & \left\langle X^{4 \widetilde{4}}\right\rangle & 0 & \left\langle X^{44}+X^{\widetilde{11}}\right\rangle
\end{array}\right) \times\left(\begin{array}{c}
\alpha_{1}^{14} \\
\alpha_{2}^{14} \\
\alpha_{3}^{14} \\
\alpha_{4}^{14}
\end{array}\right)=\boldsymbol{\Phi}_{14}
$$

with $\boldsymbol{\Phi}_{\mathbf{1 4}}$ vector, whose elements are averages of the products of the X- and $\xi$-operators.

$$
\begin{aligned}
\Phi_{14}^{1}= & V \sin \left(\phi_{4}-\phi_{1}\right)\left\langle\xi_{\uparrow} X^{\widetilde{4} 1}-X^{4 \widetilde{1}} \xi_{\uparrow}\right\rangle+V\left\langle X^{42} \xi_{\downarrow}^{+} \cos \left(\phi_{2}-\phi_{4}\right)\right. \\
& \left.+X^{\widetilde{2}} \xi_{\downarrow}^{+} \sin \left(\phi_{2}-\phi_{4}\right)\right\rangle+V\left\langle X^{31} \xi_{\downarrow} \cos \left(\phi_{3}-\phi_{1}\right)+X^{\widetilde{3} 1} \xi_{\downarrow} \sin \left(\phi_{3}-\phi_{1}\right)\right\rangle, \\
\Phi_{14}^{2}= & 0, \\
\Phi_{14}^{3}= & V\left\langle X^{\widetilde{4} 1} \xi_{\uparrow} \cos \left(\phi_{4}-\phi_{1}\right)-X^{\widetilde{4} 1} \xi_{\uparrow} \sin \left(\phi_{4}-\phi_{1}\right)\right\rangle+V\left\langle X^{\widetilde{4} 2} \xi_{\downarrow}^{+} \cos \left(\phi_{2}-\phi_{4}\right)\right\rangle \\
& +V\left\langle X^{\widetilde{42}} \xi_{\downarrow}^{+} \sin \left(\phi_{2}-\phi_{4}\right)\right\rangle, \\
\Phi_{14}^{4}= & V\left\langle X^{4 \widetilde{1}} \xi_{\uparrow} \cos \left(\phi_{4}-\phi_{1}\right)+X^{\widetilde{4} 1} \xi_{\uparrow} \sin \left(\phi_{4}-\phi_{1}\right)\right\rangle+V\left\langle X^{3 \widetilde{1}} \xi_{\downarrow} \cos \left(\phi_{3}-\phi_{1}\right)\right\rangle \\
& +V\left\langle X^{\widetilde{31}} \xi_{\downarrow} \sin \left(\phi_{3}-\phi_{1}\right)\right\rangle .
\end{aligned}
$$


In the same way one can determine the coefficients $\alpha_{5}^{14}, \alpha_{6}^{14}, \alpha_{7}^{14}, \alpha_{8}^{14}$.

Then, $Z^{14}$ operator can be written after projecting onto the basic X-operators as follows:

$$
\begin{aligned}
Z^{14}= & V \cos \left(\phi_{4}-\phi_{1}\right)\left(X^{11}+X^{44}\right) \xi_{\uparrow}+V \sin \left(\phi_{4}-\phi_{1}\right)\left(X^{\widetilde{4} 4}+X^{1 \widetilde{1}}\right) \xi_{\uparrow}+V X^{12} \xi_{\downarrow}^{+} \cos \left(\phi_{2}-\phi_{4}\right) \\
& +V X^{1 \widetilde{2}} \xi_{\downarrow}^{+} \sin \left(\phi_{2}-\phi_{4}\right)+V X^{34} \xi_{\downarrow} \cos \left(\phi_{3}-\phi_{1}\right)+V X^{\widetilde{3} 4} \xi_{\downarrow} \sin \left(\phi_{3}-\phi_{1}\right) \\
& -\alpha_{1}^{14} X^{14}-\alpha_{2}^{14} X^{\widetilde{14}}-\alpha_{3}^{14} X^{1 \widetilde{4}}-\alpha_{4}^{14} X^{\widetilde{1} 4}-\alpha_{5}^{14} X^{32}-\alpha_{6}^{14} X^{\widetilde{32}}-\alpha_{7}^{14} X^{\widetilde{3} 2}-\alpha_{8}^{14} X^{3 \widetilde{2}} .(34)
\end{aligned}
$$

In this way one can present the equations of motion for other X-operators $X^{\widetilde{14}}, X^{1 \widetilde{4}} \ldots X^{3 \widetilde{2}}$ similar to (29). Now we have:

$$
\hat{\mathbf{B}}\left(\begin{array}{l}
\left\langle\left\langle X^{14} \mid X^{41}\right\rangle\right\rangle \\
\left\langle\left\langle X^{\overline{14}} \mid X^{41}\right\rangle\right\rangle \\
\left\langle\left\langle X^{1 \overline{4}} \mid X^{41}\right\rangle\right\rangle \\
\left\langle\left\langle X^{\tilde{1} 4} \mid X^{41}\right\rangle\right\rangle \\
\left\langle\left\langle X^{32} \mid X^{41}\right\rangle\right\rangle \\
\left\langle\left\langle X^{\overline{32}} \mid X^{41}\right\rangle\right\rangle \\
\left\langle\left\langle X^{32} \mid X^{41}\right\rangle\right\rangle \\
\left\langle\left\langle X^{\tilde{3} 2} \mid X^{41}\right\rangle\right\rangle
\end{array}\right)=\left(\begin{array}{c}
\frac{\left\langle X^{11}+X^{44}\right\rangle}{2 \pi}+\left\langle\left\langle Z^{14} \mid X^{41}\right\rangle\right\rangle \\
\left\langle\left\langle Z^{\overline{14}} \mid X^{41}\right\rangle\right\rangle \\
\left\langle\left\langle Z^{1 \overline{4}} \mid X^{41}\right\rangle\right\rangle \\
\left\langle\left\langle Z^{14} \mid X^{41}\right\rangle\right\rangle \\
\left\langle\left\langle Z^{32} \mid X^{41}\right\rangle\right\rangle \\
\left\langle\left\langle Z^{\tilde{3}} \mid X^{41}\right\rangle\right\rangle \\
\left\langle\left\langle Z^{3 \tilde{2}} \mid X^{41}\right\rangle\right\rangle \\
\left\langle\left\langle Z^{\tilde{3} 2} \mid X^{41}\right\rangle\right\rangle
\end{array}\right),
$$

where

$$
\hat{\mathbf{B}}=\left(\begin{array}{ccc}
\omega-\varepsilon_{41}-\alpha_{1}^{14} & \cdots & -\alpha_{1}^{\tau^{72}} \\
-\alpha_{2}^{14} & \cdots & -\alpha_{2}^{72} \\
-\alpha_{3}^{14} & \cdots & -\alpha_{3}^{72} \\
-\alpha_{4}^{14} & \cdots & -\alpha_{4}^{72} \\
-\alpha_{5}^{14} & \cdots & -\alpha_{5}^{72} \\
-\alpha_{6}^{14} & \cdots & -\alpha_{6}^{72} \\
-\alpha_{7}^{14} & \cdots & -\alpha_{7}^{72} \\
-\alpha_{8}^{14} & \cdots & \omega-\varepsilon_{\widetilde{3} 2}-\alpha_{8}^{\widetilde{7} 2}
\end{array}\right)
$$

Equations for Green's functions $\left\langle\left\langle Z^{m n} \mid X^{41}\right\rangle\right\rangle$ can be obtained by differentiating with respect to the second time argument; for example

$$
\begin{aligned}
& \left\langle\left\langle Z^{14} \mid X^{41}\right\rangle\right\rangle\left(\omega-\varepsilon_{41}\right)=\left\langle\left\langle Z^{14} \mid X^{41}\right\rangle\right\rangle \alpha_{1}^{14}+\left\langle\left\langle Z^{14} \mid X^{\widetilde{4}}\right\rangle\right\rangle \alpha_{2}^{14}+\left\langle\left\langle Z^{14} \mid X^{1 \widetilde{4}}\right\rangle\right\rangle \alpha_{3}^{14} \\
& \quad+\left\langle\left\langle Z^{14} \mid X^{\widetilde{1} 4}\right\rangle\right\rangle \alpha_{4}^{14}+\left\langle\left\langle Z^{14} \mid X^{23}\right\rangle\right\rangle \alpha_{5}^{14}+\left\langle\left\langle Z^{14} \mid X^{\widetilde{23}}\right\rangle\right\rangle \alpha_{6}^{14}+\left\langle\left\langle Z^{14} \mid X^{\widetilde{2} 3}\right\rangle\right\rangle \alpha_{7}^{14} \\
& \quad+\left\langle\left\langle Z^{14} \mid X^{2 \widetilde{3}}\right\rangle\right\rangle \alpha_{8}^{14}+\left\langle\left\langle Z^{14} \mid Z^{41}\right\rangle\right\rangle,
\end{aligned}
$$

where $\varepsilon_{p q}=\varepsilon_{p}-\varepsilon_{q}$. Using the similar procedure we can write the equations for the functions containing other operators in the right hand part. Finally, for the matrix Green's function:

$$
\hat{\mathbf{G}}_{\uparrow}=2 \pi\left(\begin{array}{ccc}
\left\langle\left\langle X^{14} \mid X^{41}\right\rangle\right\rangle & \cdots & \left\langle\left\langle X^{14} \mid X^{2 \tilde{3}}\right\rangle\right\rangle \\
\ldots \ldots \ldots \ldots \ldots \cdots \cdots & \ldots \ldots \ldots \ldots \cdots \cdots \\
\left\langle\left\langle X^{\tilde{3} 2} \mid X^{41}\right\rangle\right\rangle & \cdots & \left\langle\left\langle X^{\widetilde{3} 2} \mid X^{2 \widetilde{3}}\right\rangle\right\rangle
\end{array}\right),
$$

we can write the following equation:

$$
\hat{\mathbf{G}}_{\uparrow}=\hat{\mathbf{G}}_{\uparrow}^{0}+\hat{\mathbf{G}}_{\uparrow}^{0} \hat{\mathbf{P}}_{\uparrow} \hat{\mathbf{G}}_{\uparrow}^{0} .
$$

Here $\hat{\mathbf{G}}_{\uparrow}^{\mathbf{0}}$ is the nonperturbed Green's function:

$$
\hat{\mathbf{G}}_{\uparrow}^{\mathbf{0}}=\hat{\mathbf{B}}^{-1} \hat{\mathbf{I}}_{\uparrow},
$$




$$
\hat{\mathbf{I}_{\uparrow}}=\left(\begin{array}{cccccccc}
A_{14}^{-1} & 0 & 0 & 0 & 0 & 0 & 0 & 0 \\
0 & A_{\tilde{14}}^{-1} & 0 & 0 & 0 & 0 & 0 & 0 \\
0 & 0 & A_{1 \overline{4}}^{-1} & 0 & 0 & 0 & 0 & 0 \\
0 & 0 & 0 & A_{\tilde{1} 4}^{-1} & 0 & 0 & 0 & 0 \\
0 & 0 & 0 & 0 & A_{32}^{-1} & 0 & 0 & 0 \\
0 & 0 & 0 & 0 & 0 & A_{\tilde{3} \tilde{2}}^{-1} & 0 & 0 \\
0 & 0 & 0 & 0 & 0 & 0 & A_{3 \tilde{2}}^{-1} & 0 \\
0 & 0 & 0 & 0 & 0 & 0 & 0 & A_{\tilde{3} 2}^{-1}
\end{array}\right),
$$

where $A_{p q}=\left\langle X^{p p}+X^{q q}\right\rangle$.

The matrix

$$
\hat{\mathbf{P}}_{\uparrow}=2 \pi \hat{\mathbf{I}_{\uparrow}}\left(\begin{array}{ccc}
\left\langle\left\langle Z^{14} \mid Z^{41}\right\rangle\right\rangle & \cdots & \left\langle\left\langle Z^{14} \mid Z^{2 \widetilde{3}}\right\rangle\right\rangle \\
\cdots \ldots \ldots \ldots \ldots \cdots \cdots & \ldots \ldots \ldots \ldots \ldots \cdots \\
\left\langle\left\langle Z^{\widetilde{3} 2} \mid Z^{41}\right\rangle\right\rangle & \cdots & \left\langle\left\langle Z^{\widetilde{3} 2} \mid Z^{2 \widetilde{3}}\right\rangle\right\rangle
\end{array}\right) \hat{\mathbf{I}_{\uparrow}}
$$

has the meaning of a scattering matrix. Being expressed in terms of irreducible Green's functions, it contains the scattering corrections of the second and higher order in powers of $V$. The separation in $\hat{P}_{\uparrow}$ of irreducible, with respect to $V$, parts enables us to obtain a mass operator $\hat{M}_{\uparrow}[9]$

$$
\hat{\mathbf{P}}_{\uparrow}=\hat{\mathbf{M}}_{\uparrow}+\hat{\mathbf{M}}_{\uparrow} \hat{\mathbf{G}}_{\uparrow}^{0} \hat{\mathbf{M}}_{\uparrow}+\hat{\mathbf{M}}_{\uparrow} \hat{\mathbf{G}}_{\uparrow}^{0} \hat{\mathbf{M}}_{\uparrow} \hat{\mathbf{G}}_{\uparrow}^{0} \hat{\mathbf{M}}_{\uparrow}+\cdots, \quad \hat{\mathbf{M}}_{\uparrow}=\left.\hat{\mathbf{P}}_{\uparrow}\right|_{\mathrm{ir}} .
$$

In this case equation (39) can be transformed into Dyson equation:

$$
\hat{\mathbf{G}}_{\uparrow}=\hat{\mathbf{G}}_{\uparrow}^{0}+\hat{\mathbf{G}}_{\uparrow}^{0} \hat{\mathbf{M}}_{\uparrow} \hat{\mathbf{G}}_{\uparrow}
$$

with the solution

$$
\hat{\mathbf{G}}_{\uparrow}=\left(1-\hat{\mathbf{G}}_{\uparrow}^{0} \hat{\mathbf{M}}_{\uparrow}\right)^{-1} \hat{\mathbf{G}}_{\uparrow}^{0}
$$

\section{Different-time decoupling of irreducible Green's functions}

To calculate the Green's functions entering the expressions for elements of matrix $\hat{\mathbf{P}}_{\uparrow}$, we shall use the method of different-time decoupling described in [12]. This procedure means in our case an independent averaging of products of $X$ and $\xi$ operators. As it was shown above, for $Z^{14}$ we have an expression (34) that can be written in the form

$$
\begin{aligned}
\frac{1}{V} Z^{14}= & \cos \left(\phi_{4}-\phi_{1}\right)\left(X^{11} \widetilde{X^{44}}\right) \xi_{\uparrow}+\widetilde{X^{12} \xi_{\downarrow}^{+}} \cos \left(\phi_{2}-\phi_{4}\right)+\sin \left(\phi_{4}-\phi_{1}\right)\left(X^{\widetilde{4} 4}+X^{1 \widetilde{1}}\right) \xi_{\uparrow} \\
& +\widetilde{X^{1 \widetilde{2}} \xi_{\downarrow}^{+}} \sin \left(\phi_{2}-\phi_{4}\right)+\widetilde{X^{34} \xi_{\downarrow}} \cos \left(\phi_{3}-\phi_{1}\right)+\widetilde{X^{\widetilde{3} 4} \xi_{\downarrow}} \sin \left(\phi_{3}-\phi_{1}\right),
\end{aligned}
$$

where in order to write the operators $\left(X^{p q+X^{r s}}\right) \xi_{\sigma}$ and $\widetilde{X^{r s} \xi_{\sigma}}$ one has to solve the equation (32) for $\alpha_{k}^{p q}$ coefficients. Let us now consider the parts of the function $\left\langle\left\langle Z^{14} \mid Z^{41}\right\rangle\right\rangle$ separately.

1. The Green's function $\left\langle\left\langle\left(X^{11+X^{44}}\right) \xi_{\uparrow} \mid \xi_{\uparrow}^{+}\left(\widetilde{X^{11}+X^{44}}\right)\right\rangle\right\rangle_{\omega} \equiv I_{1}(\omega)$.

According to the spectral theorem

$$
I_{1}(\omega)=\frac{1}{2 \pi} \int_{-\infty}^{\infty} \frac{\mathrm{d} \omega^{\prime}}{\omega-\omega^{\prime}}\left(\mathrm{e}^{\beta \omega^{\prime}}+1\right) \int_{-\infty}^{\infty} \frac{\mathrm{d} t}{2 \pi} \mathrm{e}^{-\mathrm{i} \omega^{\prime} t}\left\langle\xi_{\uparrow}^{+}(t)\left(X^{11}+X^{44}\right)_{t}\left(X^{11}+X^{44}\right) \xi_{\uparrow}\right\rangle^{\mathrm{ir}} .
$$

Due to the different-time decoupling and after taking the averages in these correlators in the zero-approximation

$$
\begin{aligned}
\left\langle\xi_{\uparrow}^{+}(t)\left(X^{11}+X^{44}\right)_{t}\left(X^{11}+X^{44}\right) \xi_{\uparrow}\right\rangle^{\text {ir }} & \approx\left\langle\left(X^{11}+X^{44}\right)_{t}\left(X^{11}+X^{44}\right)\right\rangle\left\langle\xi_{\uparrow}^{+}(t) \xi_{\uparrow}\right\rangle, \\
\left\langle\left(X^{11}+X^{44}\right)_{t}\left(X^{11}+X^{44}\right)\right\rangle & \approx\left\langle\left(X^{11}+X^{44}\right)^{2}\right\rangle=A_{14} .
\end{aligned}
$$


This leads to the result:

$$
I_{1}(\omega)=A_{14}\left\langle\left\langle\xi_{\uparrow} \mid \xi_{\uparrow}^{+}\right\rangle\right\rangle
$$

2. The Green's function $\left\langle\left\langle\left(X^{1 \widetilde{1}+X^{\widetilde{4}} 4}\right) \xi_{\uparrow} \mid \xi_{\uparrow}^{+}\left(\widetilde{X^{\widetilde{1} 1}+X^{4 \widetilde{4}}}\right)\right\rangle\right\rangle_{\omega} \equiv I_{2}(\omega)$ :

$$
\begin{aligned}
\left\langle\xi_{\uparrow}^{+}(t)\left(X^{\tilde{1} 1}+X^{4 \widetilde{4}}\right)_{t}\left(X^{1 \tilde{1}}+X^{\widetilde{4} 4}\right) \xi_{\uparrow}\right\rangle^{\mathrm{ir}} & \approx\left\langle\left(X^{\widetilde{1} 1}+X^{4 \widetilde{4}}\right)_{t}\left(X^{1 \widetilde{1}}+X^{\widetilde{4} 4}\right)\right\rangle\left\langle\xi_{\uparrow}^{+}(t) \xi_{\uparrow}\right\rangle, \\
\left\langle\left(X^{\tilde{1} 1}+X^{4 \widetilde{4}}\right)_{t}\left(X^{1 \tilde{1}}+X^{\widetilde{4} 4}\right)\right\rangle & =\left\langle X_{t}^{\tilde{1} 1} X^{1 \tilde{1}}+X_{t}^{4 \widetilde{4}} X^{\widetilde{4} 4}\right\rangle \\
& =\exp \left[\mathrm{i}\left(\varepsilon_{\tilde{1}}-\varepsilon_{1}\right) t\right]\left\langle X^{\widetilde{11}}\right\rangle+\exp \left[\mathrm{i}\left(\varepsilon_{4}-\varepsilon_{\tilde{4}}\right) t\right]\left\langle X^{44}\right\rangle .
\end{aligned}
$$

Using this approximation we have:

$$
\begin{aligned}
I_{2}(\omega) & =\frac{1}{2}\left\langle X^{44}+X^{\widetilde{44}}\right\rangle\left\langle\left\langle\xi_{\uparrow} \mid \xi_{\uparrow}^{+}\right\rangle\right\rangle_{\omega-\varepsilon_{4}+\varepsilon_{\widetilde{4}}}+\frac{1}{2}\left\langle X^{11}+X^{\widetilde{11}}\right\rangle\left\langle\left\langle\xi_{\uparrow} \mid \xi_{\uparrow}^{+}\right\rangle\right\rangle_{\omega-\varepsilon_{\tilde{1}}+\varepsilon_{1}}+\frac{1}{2 \pi}\left\langle X^{\widetilde{44}}-X^{44}\right\rangle \\
& \times \int_{-\infty}^{\infty} \frac{\operatorname{th} \frac{\beta \omega^{\prime}}{2}\left[-2 \operatorname{Im}\left\langle\left\langle\xi_{\uparrow} \mid \xi_{\uparrow}^{+}\right\rangle\right\rangle_{\omega^{\prime}+\mathrm{i} \delta}\right] \mathrm{d} \omega^{\prime}}{\omega-\omega^{\prime}-\varepsilon_{4}+\varepsilon_{\widetilde{4}}}+\frac{1}{2 \pi}\left\langle X^{11}-X^{\widetilde{11}}\right\rangle \int_{-\infty}^{\infty} \frac{\operatorname{th} \frac{\beta \omega^{\prime}}{2}\left[-2 \operatorname{Im}\left\langle\left\langle\xi_{\uparrow} \mid \xi_{\uparrow}^{+}\right\rangle\right\rangle_{\omega^{\prime}+i \delta}\right] \mathrm{d} \omega^{\prime}}{\omega-\omega^{\prime}-\varepsilon_{\tilde{1}}+\varepsilon_{1}} .
\end{aligned}
$$

In a similar way we calculate the Green's function $\left.\left\langle\left\langle\widetilde{\left\langle X^{12} \xi_{\downarrow}^{+}\right.} \mid \widetilde{\xi_{\downarrow} X^{21}}\right\rangle\right\rangle,\left\langle\widehat{\left\langle X^{34} \xi_{\downarrow}\right.} \mid \widetilde{\xi_{\downarrow}^{+} X^{43}}\right\rangle\right\rangle$, etc. (see Appendix)

\section{Two near subbands in alloy analogy approximation}

The scattering processes are taken here into account only via coherent potential. This corresponds to the neglect of averages $\left\langle X^{m n} \xi_{\sigma}\right\rangle[5,9]$ and to the neglect of terms $R_{(1,2) \uparrow}^{p q}(\omega)$ in expressions for irreducible Green's functions. The matrix of nonperturbed single-site Green's function is diagonal in AA approximation:

$$
\begin{aligned}
& \hat{\mathbf{G}}_{\uparrow}^{\mathbf{0}}=\left(\begin{array}{cc}
\hat{\mathbf{G}}_{\uparrow}^{\mathbf{0}}(14 \ldots 4 \widetilde{1}) & 0 \\
0 & \hat{\mathbf{G}}_{\uparrow}^{\mathbf{0}}(32 \ldots 2 \widetilde{3})
\end{array}\right) \\
& \hat{\mathbf{G}}_{\uparrow}^{\mathbf{0}}(14 \ldots 4 \widetilde{1})=\left(\begin{array}{cccc}
\frac{A_{14}}{\omega-\varepsilon_{41}} & 0 & 0 & 0 \\
0 & \frac{A_{\widetilde{1}}}{\omega-\varepsilon_{\widetilde{1}}} & 0 & 0 \\
0 & 0 & \frac{A_{1 \widetilde{4}}}{\omega-\varepsilon_{\tilde{4} 1}} & 0 \\
0 & 0 & 0 & \frac{A_{\widetilde{1} 4}}{\omega-\varepsilon_{4 \widetilde{1}}}
\end{array}\right) \text {, } \\
& \hat{\mathbf{G}}_{\uparrow}^{\mathbf{0}}(32 \ldots 2 \widetilde{3})=\left(\begin{array}{cccc}
\frac{A_{32}}{\omega-\varepsilon_{23}} & 0 & 0 & 0 \\
0 & \frac{A_{\widetilde{3} \widetilde{2}}}{\omega-\varepsilon_{\tilde{2} \tilde{3}}} & 0 & 0 \\
0 & 0 & \frac{A_{3 \widetilde{2}}}{\omega-\varepsilon_{\tilde{2} 3}} & 0 \\
0 & 0 & 0 & \frac{A_{\widetilde{3} 2}}{\omega-\varepsilon_{2 \widetilde{3}}}
\end{array}\right) \text {. }
\end{aligned}
$$

The matrix of the mass operator in this case is:

$$
\hat{\mathbf{M}}_{\uparrow}=\left(\begin{array}{cc}
\hat{\mathbf{M}}_{\uparrow}(14 \ldots 4 \widetilde{1}) & 0 \\
0 & \hat{\mathbf{M}}_{\uparrow}(32 \ldots 2 \widetilde{3})
\end{array}\right)
$$




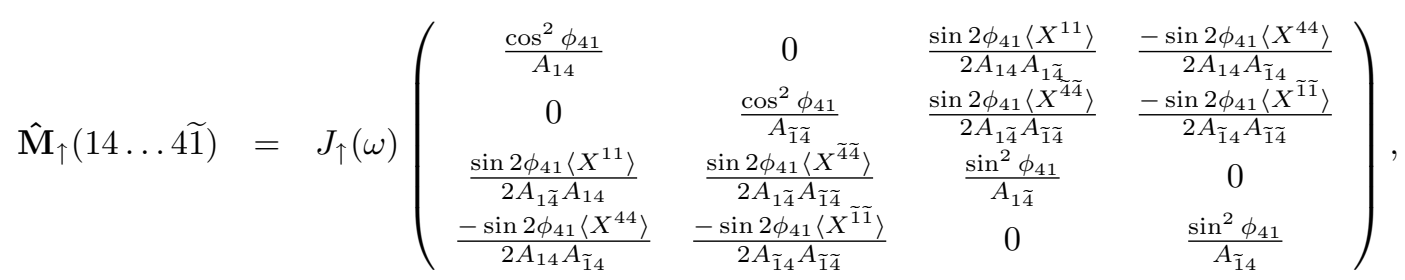

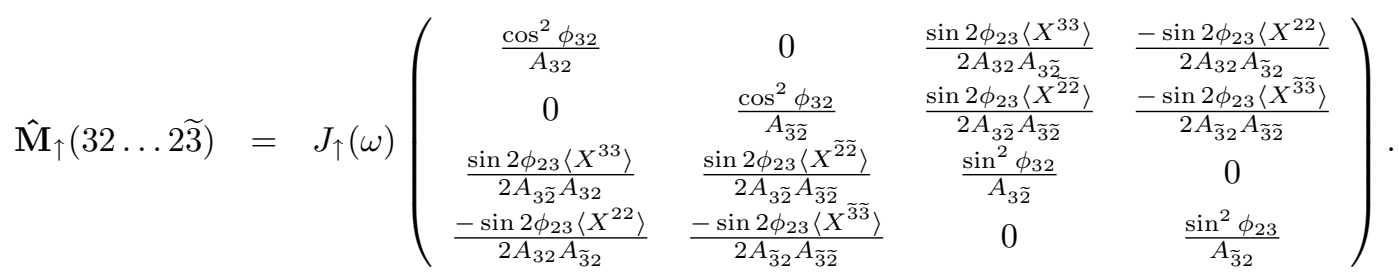

Respectively, the Green's function matrix as well as the mass operator matrix consist of two independent blocks:

$$
\hat{\mathbf{G}}_{\uparrow}=\left(\begin{array}{cc}
\hat{\mathbf{G}}_{\uparrow}(14 \ldots 4 \widetilde{1}) & 0 \\
0 & \hat{\mathbf{G}}_{\uparrow}(32 \ldots 2 \widetilde{3})
\end{array}\right) .
$$

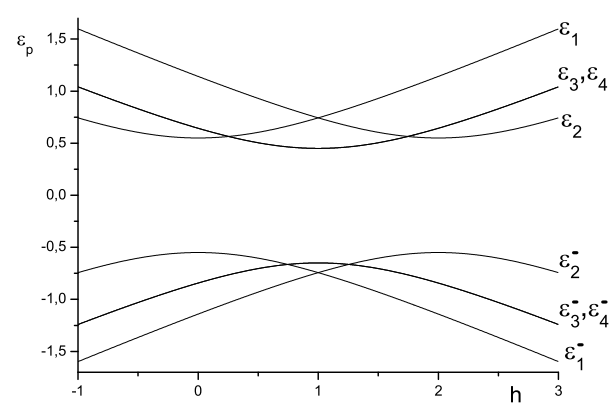

Figure 1. Single-site electron levels $\varepsilon_{p}$ as functions of $h$ at $\Omega=1.1 . n=1.0, g=$ $1.0, U=0.2$.

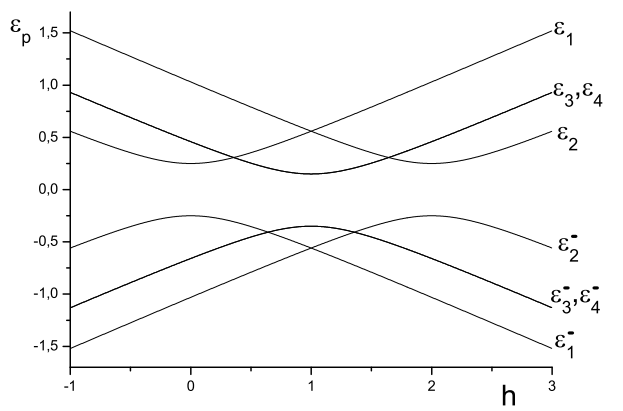

Figure 2. Single-site electron levels $\varepsilon_{p}$ as functions of $h$ at $\Omega=0.5 . n=1.0, g=$ $1.0, U=0.2$.

Using equation (44) and formulae (52) we obtain the components of matrices $\hat{\mathbf{G}}_{\uparrow}(14 \ldots \widetilde{4} \widetilde{1})$ and $\hat{\mathbf{G}}_{\uparrow}(32 \ldots \widetilde{23})$. Substituting them into relation (26) we get the single-site Green's function $\hat{\mathbf{G}}_{\uparrow}^{(\mathbf{a})}(\omega)$ in the final form. In general, the obtained expression is rather complicated. Contributions from individual electron transitions with energies $\varepsilon_{p q}$ between initial single-site levels $|p\rangle$ and $|q\rangle$ depend on their position in the spectrum. The latter are sensitive to values of the $h, \Omega$ and $U$ parameters and are also determined by averages $A_{p q}=\left\langle X^{p p}+X^{q q}\right\rangle$, where in the zero approximation $\left\langle X^{p p}\right\rangle \sim$ $\exp \left(-\beta \varepsilon_{p}\right)$ (it means that at low temperatures only lower energy levels should be considered). The dependence of single-site electron levels on asymmetry field $h$ at different values of the tunneling splitting parameter $\Omega$ is shown in figures 1,2 . In this case only the levels with negative pseudospin projection $\varepsilon_{\tilde{1}} \ldots \varepsilon_{\widetilde{4}}$ are important. Energies of electron transitions between the levels in figure 2 are shown in figure 3. At certain values of the field $h$ some pairs of electron transitions become close by their energy (respectively, there appear the near subbands in the resulting electron spectrum). At $h \sim 0$ such is the case for pair of transitions $\widetilde{23}$ and $\widetilde{4} \widetilde{1}$; the chemical potential of electrons is placed in that region at concentrations near half-filling, $n \sim 1$ [7] In this case very important is the parameter $\Delta=\varepsilon_{\widetilde{2} \widetilde{3}}-\varepsilon_{\widetilde{4} \widetilde{1}}$, which has the meaning of the effective on-site interaction energy of electrons (more precisely, the energy of the appearance of the "excess electron"-"hole" pair) in 
the presence of interaction with pseudospins [13]. At $\Delta<0$ the transition energies change their relative position that corresponds to the effective attraction. In figure 4 a diagram is shown, where such a region is shown on the $(\Omega, U)$ plane at different values of $h$. The subbands $(\widetilde{14})$ and $(\widetilde{32})$ are close and can overlap when $\Delta \lesssim W$, where $W$ is the half-width of the initial electron band $(W<g, U)$. It is the case which is considered further.

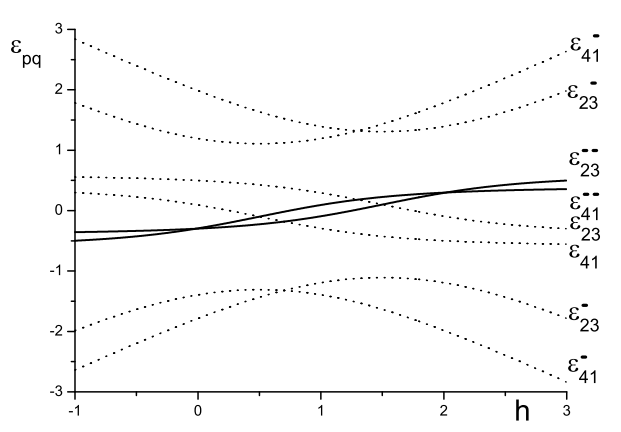

Figure 3. Energies of single-site electron levels transitions $\varepsilon_{p q}=\varepsilon_{p}-\varepsilon_{q}$ as function of $h . n=1.0, g=1.0, U=0.2, \Omega=0.5$.

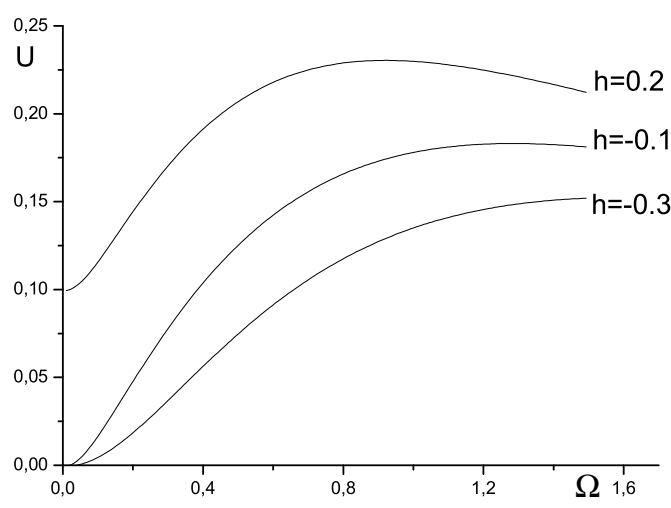

Figure 4. Diagram $\Delta=0$. In the region below the curve, the parameter $\Delta$ is negative.

The Green's function $G_{\uparrow}^{(a)}(\omega)$ for this problem when the subbands $(\widetilde{14})$ and $(\widetilde{3} \widetilde{2})$ interplay with each other is as follows:

$$
\begin{aligned}
G_{\uparrow}^{(a)}(\omega) & =\left\langle\left\langle X^{\widetilde{1} \widetilde{4}} \mid X^{\widetilde{4} \widetilde{1}}\right\rangle\right\rangle+\left\langle\left\langle X^{\widetilde{32}} \mid X^{\widetilde{2} \widetilde{3}}\right\rangle\right\rangle \\
& =\frac{\left\langle X^{\widetilde{11}}+X^{\widetilde{4}}\right\rangle \cos ^{2} \phi_{41}}{\omega-\varepsilon_{\widetilde{4} \widetilde{1}}-J_{\uparrow} \cos ^{2}\left(\phi_{4}-\phi_{1}\right)}+\frac{\left\langle X^{\widetilde{3} \widetilde{3}}+X^{\widetilde{2}}\right\rangle \cos ^{2} \phi_{32}}{\omega-\varepsilon_{\widetilde{2} \widetilde{3}}-J_{\uparrow} \cos ^{2}\left(\phi_{3}-\phi_{2}\right)} .
\end{aligned}
$$

This is a particular case of equation (20) for two near subbands in alloy analogy approximation. Now, using property of the locality of $\Xi_{\sigma}(\omega)$ function and equations (18) and (19), we can connect the Green's function $G_{\uparrow}^{(a)}(\omega)$ and the coherent potential $J_{\uparrow}(\omega)$ as follows:

$$
G_{\uparrow}^{(a)}(\omega)=\frac{1}{N} \sum_{\vec{k}} \frac{1}{G_{\uparrow}^{(a)}(\omega)+J_{\uparrow}(\omega)-t_{k}}=\int_{-\infty}^{\infty} \mathrm{d} t \frac{\rho_{0}(t)}{G_{\uparrow}^{(a)}(\omega)+J_{\uparrow}(\omega)-t_{k}} .
$$

Excluding with the help of $(54)$ the Green's function $G_{\uparrow}(\omega)$ for Bethe lattice with semi-elliptical density of states $\rho_{0}(t)=\frac{2}{\pi W^{2}} \sqrt{W^{2}-t^{2}}$, we have

$$
J_{\uparrow}=\frac{W^{2}}{4}\left[\frac{\left\langle X^{\widetilde{1} \widetilde{1}}+X^{\widetilde{4}}\right\rangle \cos ^{2} \phi_{41}}{\omega-\varepsilon_{\widetilde{4} \tilde{1}}-J_{\uparrow} \cos ^{2} \phi_{41}}+\frac{\left\langle X^{\widetilde{3}}+X^{\widetilde{2} \widetilde{2}}\right\rangle \cos ^{2} \phi_{32}}{\omega-\varepsilon_{\widetilde{2} \widetilde{3}}-J_{\uparrow} \cos ^{2} \phi_{32}}\right] .
$$

We can also take into account that in the considered case:

$$
\left\langle X^{\widetilde{1} \widetilde{1}}+X^{\widetilde{4} \widetilde{4}}\right\rangle \cong 1-\frac{n}{2}, \quad\left\langle X^{\widetilde{33}}+X^{\widetilde{22}}\right\rangle \cong \frac{n}{2}
$$

and equation for the chemical potential has the form

$$
\left\langle X^{\widetilde{4} \widetilde{4}}\right\rangle=\left\langle X^{\widetilde{3} \widetilde{3}}\right\rangle
$$

where the averages of X-operators can be calculated using the spectral theorem for the corresponding components of Green's function (53). One can obtain the chemical potential and determine the edges of subbands as functions of the average electron concentration when the (56), 
(58) are solved. In the limit $T \rightarrow 0,(\beta \rightarrow \infty)$ the chemical potential dependence on $n$ is shown in figure 5 ; the case is considered when two near subbands $\widetilde{14}$ and $\widetilde{32}$ do not overlap. The possibility of two separate subbands existing or joining a single one (when a gap in spectrum disappears) depends on the model parameter values. Figure 6 shows the both examples. There exists the critical value $U_{\text {eff }}$ of the on-site interaction parameter at which the resulting density of states $\rho(\omega)$ changes its topology (the gap exists at $U>U_{\text {eff }}$ ). The dependencies $U_{\text {eff }}$ on the asymmetry field and the tunneling level splitting are shown in figures 7 and 8 , respectively.

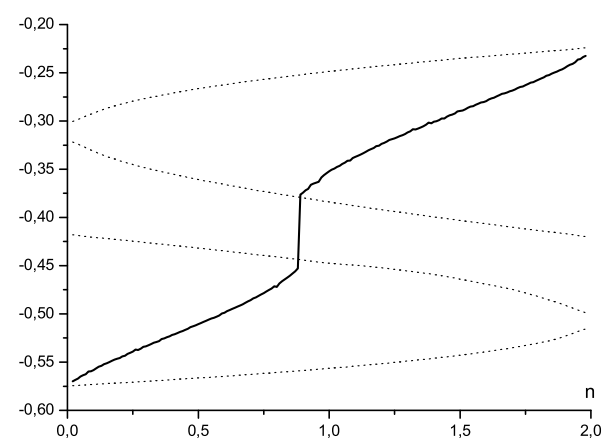

Figure 5. Dependence of chemical potential and edges of subbands $(\widetilde{1} \widetilde{4})$ and $\widetilde{3} \widetilde{2}$ on $n$ at zero temperature. The subband $(\widetilde{1} \widetilde{4})$ is the lower one. $g=1.0, W=0.1, U=0.3, \Omega=0.5, h=-0.1$.

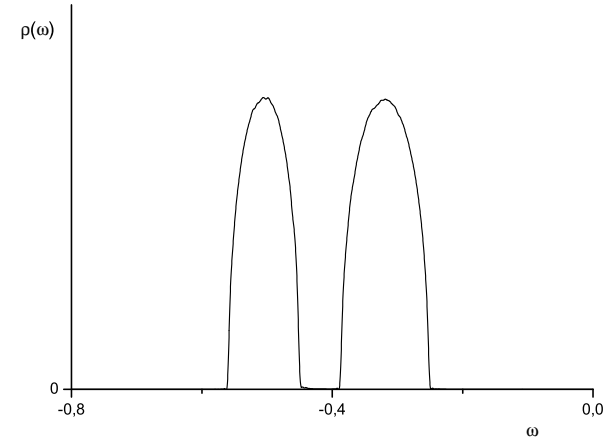

(a)

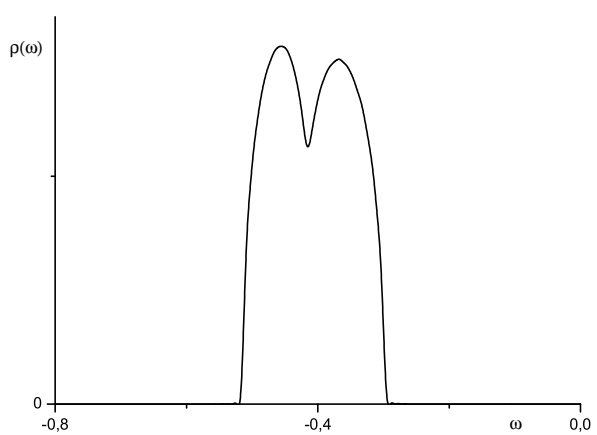

(b)

Figure 6. Density of states $\rho(\omega)$ at various values of the on-site interaction parameter $U . U=0.3$ : two separated subbands, $U=0.2$ : the one common subband. $\Omega=0.5, h=-0.1, n=1.0$.

We can also determine the critical value of the tunneling splitting parameter. As function of the asymmetry field $h$, it is shown in figure 9 at different values of $U$. The gap in the spectrum opens when $\Omega$ is less than the critical one.

\section{Conclusions}

The electron energy spectrum of the pseudospin-electron model allowing for interaction of the near energy subbands is considered. For this purpose the dynamical mean field method is applied. The effective single-site problem is solved within the auxiliary fermion field approach using the procedure of different-time decoupling of the higher order Green's functions; the alloy analogy approximation is used. 


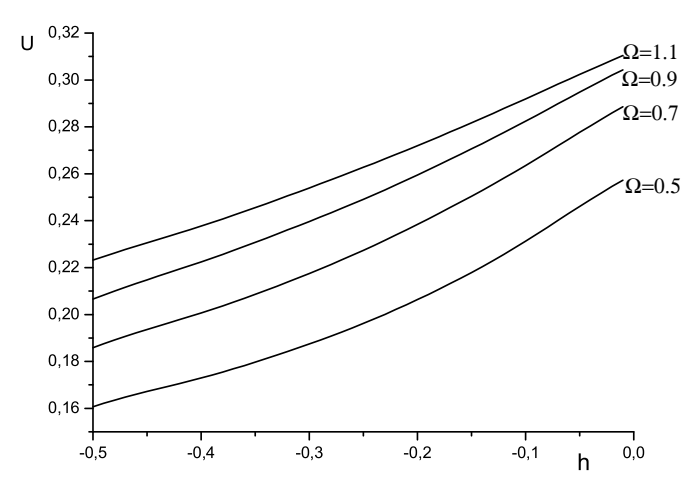

Figure 7. Dependence of the critical Coulomb potential $U_{\text {eff }}$ on the asymmetry field $h$ at different values of the tunneling splitting $\Omega ; n=1.0, g=1.0, W=0.1$.

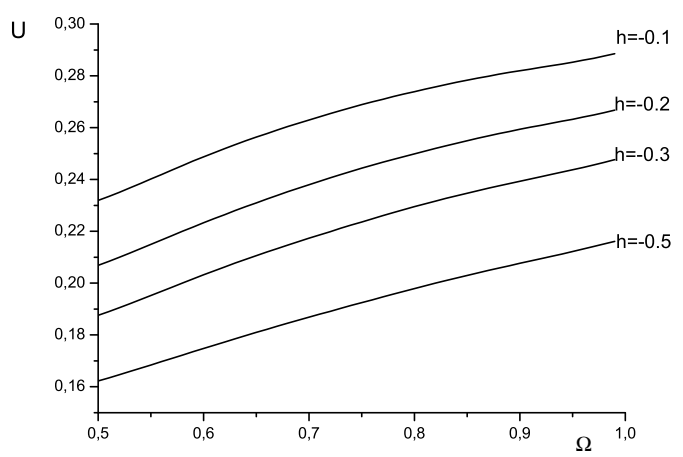

Figure 8. Dependence of the critical Coulomb potential $U_{\text {eff }}$ on the tunneling splitting $\Omega$ at different values of the asymmetry field $h n=1.0, g=1.0, W=0.1$.

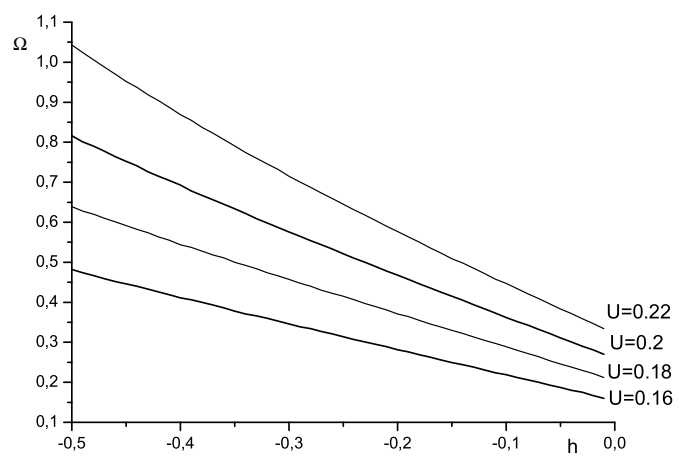

Figure 9. The critical tunneling splitting parameter $\Omega$ as the function of the asymmetry field $h$ at different values of $U ; n=1.0, g=1.0, W=0.1$.

A special case of two near subbands ( $\widetilde{14})$ and $(\widetilde{32})$ is considered. It is realized at $h \sim 0$ and is of interest when chemical potential of electrons is placed in this region (which takes place at occupying the near half-filling). Such a problem is similar to the standard Hubbard model but in our case the characteristic constants of the single-site spectrum and the electron transfer are functions of the original model parameters (field $h$, tunneling constant $\Omega$, interaction constant $g$ ). Thus, the critical value $U_{\text {crit }}$, at which a gap opens in the spectrum, depends on $\Omega$ and $h$. In the considered region of the model parameter values $U_{\text {crit }}$ increase at the increase of $\Omega$ and at the decrease of $h$ in absolute value.

The obtained results show that in the real system, which exhibits a local anharmonicity of lattice vibrations, the metal-insulator transition, determined by the short-ranged electron correlation, is effected by that anharmonic subsystem. Changing the parameters of local anharmonicity (e.g, the shape of potential well), one can affect the conditions of the gap appearance. Such an effect can be related to the mechanisms of the external pressure effect on the above mentioned metal-insulator transition in the crystalline systems with the transition and rare-earth elements having an essential lattice anharmonicity. 


\section{A. Irreducible Green's functions (different time decoupling)}

The Green's function $\left\langle\left\langle\widehat{X^{12} \xi_{\downarrow}^{+}} \mid \widetilde{\xi_{\downarrow} X^{21}}\right\rangle\right\rangle \equiv I_{3}(\omega)$ :

$$
\begin{aligned}
\left\langle\xi_{\downarrow}(t) X^{21}(t) X^{12} \xi_{\downarrow}^{+}\right\rangle^{\text {ir }} & \approx\left\langle X^{21}(t) X^{12}\right\rangle\left\langle\xi_{\downarrow}(t) \xi_{\downarrow}^{+}\right\rangle, \\
\left\langle X^{21}(t) X^{12}\right\rangle= & \exp \left[\mathrm{i}\left(\varepsilon_{2}-\varepsilon_{1}\right) t\right]\left\langle X^{22}\right\rangle, \\
I_{3}(\omega)= & \frac{1}{2}\left\langle X^{11}+X^{22}\right\rangle\left\langle\left\langle\xi_{\downarrow}^{+} \mid \xi_{\downarrow}\right\rangle\right\rangle_{\omega-\varepsilon_{2}+\varepsilon_{1}}+\frac{1}{2 \pi}\left\langle X^{11}-X^{22}\right\rangle \\
& \times \int_{-\infty}^{\infty} \frac{\operatorname{th} \frac{\beta \omega^{\prime}}{2}\left[-2 \operatorname{Im}\left\langle\left\langle\xi_{\downarrow}^{+} \mid \xi_{\downarrow}\right\rangle\right\rangle_{\omega^{\prime}+i \delta}\right] \mathrm{d} \omega^{\prime}}{\omega-\omega^{\prime}-\varepsilon_{2}+\varepsilon_{1}} .
\end{aligned}
$$

The Green's function $\left\langle\left\langle\widetilde{X^{34} \xi_{\downarrow}} \mid \widetilde{\xi_{\downarrow}^{+} X^{43}}\right\rangle\right\rangle \equiv I_{4}(\omega)$ :

$$
\begin{aligned}
\left\langle\xi_{\downarrow}^{+}(t) X^{43}(t) X^{34} \xi_{\downarrow}\right\rangle^{\text {ir }} & \approx\left\langle X^{43}(t) X^{34}\right\rangle\left\langle\xi_{\downarrow}(t) \xi_{\downarrow}^{+}\right\rangle, \\
\left\langle X^{43}(t) X^{34}\right\rangle & \approx\left\langle X^{44}\right\rangle, \\
I_{4}(\omega) & =\left\langle X^{44}\right\rangle\left\langle\left\langle\xi_{\downarrow} \mid \xi_{\downarrow}^{+}\right\rangle\right\rangle_{\omega} .
\end{aligned}
$$

The Green's function $\left\langle\left\langle\widetilde{X^{1 \widetilde{2}} \xi_{\downarrow}^{+}} \mid \widetilde{\xi_{\downarrow} X^{\widetilde{2} 1}}\right\rangle\right\rangle \equiv I_{5}(\omega)$ :

$$
\begin{aligned}
\left\langle\xi_{\downarrow}(t) X^{\widetilde{2} 1}(t) X^{1 \widetilde{2}} \xi_{\downarrow}^{+}\right\rangle^{\mathrm{ir}} \approx & \left\langle X^{\widetilde{2} 1}(t) X^{1 \widetilde{2}}\right\rangle\left\langle\xi_{\downarrow}(t) \xi_{\downarrow}^{+}\right\rangle, \\
\left\langle X^{\widetilde{2} 1}(t) X^{1 \widetilde{2}}\right\rangle= & \exp \left[\mathrm{i}\left(\varepsilon_{\tilde{2}}-\varepsilon_{1}\right) t\right]\left\langle X^{\widetilde{22}}\right\rangle, \\
I_{5}(\omega)= & \frac{1}{2}\left\langle X^{11}+X^{\widetilde{2}}\right\rangle\left\langle\left\langle\xi_{\downarrow}^{+} \mid \xi_{\downarrow}\right\rangle\right\rangle_{\omega-\varepsilon_{\tilde{2}}+\varepsilon_{1}}+\frac{1}{2 \pi}\left\langle X^{11}-X^{\widetilde{22}}\right\rangle \\
& \times \int_{-\infty}^{\infty} \frac{\operatorname{th} \frac{\beta \omega^{\prime}}{2}\left[-2 \operatorname{Im}\left\langle\left\langle\xi_{\downarrow}^{+} \mid \xi_{\downarrow}\right\rangle\right\rangle_{\omega^{\prime}+i \delta}\right] \mathrm{d} \omega^{\prime}}{\omega-\omega^{\prime}-\varepsilon_{\tilde{2}}+\varepsilon_{1}} .
\end{aligned}
$$

The Green's function $\left\langle\left\langle\widetilde{X^{\widetilde{3} 4} \xi_{\downarrow}} \mid \widetilde{\xi_{\downarrow}^{+} X^{4 \widetilde{3}}}\right\rangle\right\rangle \equiv I_{6}(\omega)$ :

$$
\begin{aligned}
\left\langle\xi_{\downarrow}^{+}(t) X^{4 \widetilde{3}}(t) X^{\widetilde{3} 4} \xi_{\downarrow}\right\rangle^{\mathrm{ir}} & =\left\langle X^{4 \widetilde{3}}(t) X^{3 \widetilde{4}}\right\rangle\left\langle\xi_{\downarrow}^{+}(t) \xi_{\downarrow}\right\rangle, \\
\left\langle X^{4 \widetilde{3}}(t) X^{\widetilde{3} 4}\right\rangle= & \exp \left[\mathrm{i}\left(\varepsilon_{4}-\varepsilon_{\widetilde{4}}\right) t\right]\left\langle X^{44}\right\rangle, \\
I_{6}(\omega)= & \frac{1}{2}\left\langle X^{44}+X^{\widetilde{3} \widetilde{3}}\right\rangle\left\langle\left\langle\xi_{\downarrow} \mid \xi_{\downarrow}^{+}\right\rangle\right\rangle_{\omega-\varepsilon_{4}+\varepsilon_{\widetilde{3}}}+\frac{1}{2 \pi}\left\langle X^{44}-X^{\widetilde{3} \widetilde{3}}\right\rangle \\
& \times \int_{-\infty}^{\infty} \frac{\operatorname{th} \frac{\beta \omega^{\prime}}{2}\left[-2 \operatorname{Im}\left\langle\left\langle\xi_{\downarrow} \mid \xi_{\downarrow}^{+}\right\rangle\right\rangle_{\omega^{\prime}+\mathrm{i} \delta}\right] \mathrm{d} \omega^{\prime}}{\omega-\omega^{\prime}-\varepsilon_{4}+\varepsilon_{\widetilde{3}}} .
\end{aligned}
$$

The full expression for the irreducible Green's function $\left\langle\left\langle Z^{14} \mid Z^{41}\right\rangle\right\rangle$ has the form

$$
\left\langle\left\langle Z^{14} \mid Z^{41}\right\rangle\right\rangle \frac{1}{V^{2}}=\cos ^{2}\left(\phi_{4}-\phi_{1}\right) A_{14}\left\langle\left\langle\xi_{\uparrow} \mid \xi_{\uparrow}^{+}\right\rangle\right\rangle+R_{(1) \uparrow}^{14}(\omega)+R_{(2) \uparrow}^{14}(\omega) .
$$

Here for $R_{(1) \uparrow}^{14}(\omega)$ we have:

$$
\begin{aligned}
R_{(1) \uparrow}^{14}(\omega)= & \frac{1}{2} \sin ^{2}\left(\phi_{4}-\phi_{1}\right) A_{4 \widetilde{4}}\left\langle\left\langle\xi_{\uparrow} \mid \xi_{\uparrow}^{+}\right\rangle\right\rangle_{\omega-\varepsilon_{4}+\varepsilon_{\widetilde{4}}}+\frac{1}{2} \sin ^{2}\left(\phi_{4}-\phi_{1}\right) A_{1 \widetilde{1}}\left\langle\left\langle\xi_{\uparrow} \mid \xi_{\uparrow}^{+}\right\rangle\right\rangle_{\omega-\varepsilon_{\tilde{1}}+\varepsilon_{1}} \\
& +\frac{1}{2} \cos ^{2}\left(\phi_{2}-\phi_{4}\right) A_{12}\left\langle\left\langle\xi_{\downarrow}^{+} \mid \xi_{\downarrow}\right\rangle\right\rangle_{\omega-\varepsilon_{2}+\varepsilon_{1}}+\left\langle X^{44}\right\rangle \cos ^{2}\left(\phi_{3}-\phi_{1}\right)\left\langle\left\langle\xi_{\downarrow} \mid \xi_{\downarrow}^{+}\right\rangle\right\rangle_{\omega} \\
& +\frac{1}{2} \sin ^{2}\left(\phi_{2}-\phi_{4}\right)\left\langle X^{11}+X^{\widetilde{2} \widetilde{2}}\right\rangle\left\langle\left\langle\xi_{\downarrow}^{+} \mid \xi_{\downarrow}\right\rangle\right\rangle_{\omega-\varepsilon_{\widetilde{2}}+\varepsilon_{1}} \\
& +\frac{1}{2} \sin ^{2}\left(\phi_{3}-\phi_{1}\right)\left\langle X^{44}+X^{\widetilde{33}}\right\rangle\left\langle\left\langle\xi_{\downarrow} \mid \xi_{\downarrow}^{+}\right\rangle\right\rangle_{\omega-\varepsilon_{4}+\varepsilon_{\widetilde{3}}}
\end{aligned}
$$


and this term takes into account a self-consistent renormalization of the single-site spectrum due to the internal dynamic fields formed by the magnon-like and electron pairs (holes) excitations as well as by pseudospin subsystem.

The last term $R_{(2) \uparrow}^{14}(\omega)$ is equal to:

$$
\begin{aligned}
R_{(2) \uparrow}^{14}(\omega)= & \frac{1}{2 \pi} \sin ^{2} \phi_{41}\left\langle X^{\widetilde{44}}-X^{44}\right\rangle \int_{-\infty}^{\infty} \frac{\operatorname{th} \frac{\beta \omega^{\prime}}{2}\left[-2 \operatorname{Im}\left\langle\left\langle\xi_{\uparrow} \mid \xi_{\uparrow}^{+}\right\rangle\right\rangle_{\omega^{\prime}+\mathrm{i} \delta}\right] \mathrm{d} \omega^{\prime}}{\omega-\omega^{\prime}-\varepsilon_{4}+\varepsilon_{\widetilde{4}}} \\
& +\frac{1}{2 \pi} \sin ^{2} \phi_{41}\left\langle X^{11}-X^{\widetilde{11}}\right\rangle \int_{-\infty}^{\infty} \frac{\operatorname{th} \frac{\beta \omega^{\prime}}{2}\left[-2 \operatorname{Im}\left\langle\left\langle\xi_{\uparrow} \mid \xi_{\uparrow}^{+}\right\rangle\right\rangle_{\omega^{\prime}+\mathrm{i} \delta}\right] \mathrm{d} \omega^{\prime}}{\omega-\omega^{\prime}-\varepsilon_{\widetilde{1}}+\varepsilon_{1}} \\
& +\frac{1}{2 \pi} \cos ^{2} \phi_{24}\left\langle X^{11}-X^{22}\right\rangle \int_{-\infty}^{\infty} \frac{\operatorname{th} \frac{\beta \omega^{\prime}}{2}\left[-2 \operatorname{Im}\left\langle\left\langle\xi_{\downarrow}^{+} \mid \xi_{\downarrow}\right\rangle\right\rangle_{\omega^{\prime}+\mathrm{i} \delta}\right] \mathrm{d} \omega^{\prime}}{\omega-\omega^{\prime}-\varepsilon_{2}+\varepsilon_{1}} \\
& +\frac{1}{2 \pi} \sin ^{2} \phi_{24}\left\langle X^{11}-X^{\widetilde{22}}\right\rangle \int_{-\infty}^{\infty} \frac{\operatorname{th} \frac{\beta \omega^{\prime}}{2}\left[-2 \operatorname{Im}\left\langle\left\langle\xi_{\downarrow}^{+} \mid \xi_{\downarrow}\right\rangle\right\rangle_{\omega^{\prime}+\mathrm{i} \delta}\right] \mathrm{d} \omega^{\prime}}{\omega-\omega^{\prime}-\varepsilon_{\widetilde{2}}+\varepsilon_{1}} \\
& +\frac{1}{2 \pi} \sin ^{2} \phi_{31}\left\langle X^{44}-X^{\widetilde{33}}\right\rangle \int_{-\infty}^{\infty} \frac{\operatorname{th} \frac{\beta \omega^{\prime}}{2}\left[-2 \operatorname{Im}\left\langle\left\langle\xi_{\downarrow} \mid \xi_{\downarrow}^{+}\right\rangle\right\rangle_{\omega^{\prime}+\mathrm{i} \delta}\right] \mathrm{d} \omega^{\prime}}{\omega-\omega^{\prime}-\varepsilon_{4}+\varepsilon_{\widetilde{3}}}
\end{aligned}
$$

and this term is responsible for scattering processes.

Terms of the $R_{(1) \uparrow}^{14}(\omega)$ and $R_{(2) \uparrow}^{14}(\omega)$ type are taken into consideration in different ways in various approximations. For example, in Hubbard-III approximation only the first of them is included $[5,9]$. In this work we use the alloy analogy approximation; in this case terms $(64,65)$ are neglected (see [9]). 


\title{
References
}

1. Stasyuk I.V. In highlights in Condensed Matter Physics, 695, 281.

2. Metzner W., Vollhardt D., Phys. Rev. Lett., 1989, 62, 260.

3. Metzner W., Phys. Rev. B., 1991, 43, 8549.

4. Müller-Hartmann E. Z. Phys. B, 1989, 74, 507.

5. Potthoff M., Herrmann T., Wegner T., Nolting W. Phys. Stat. Sol. (b), 1998, 210, 199.

6. Stasyuk I.V., Shvaika A.M., Acta Physica Polonica A, 1993, 84, No. 2, 293.

7. Stasyuk I.V., Shvaika A.M. Preprint Inst. Cond. Matt. Phys. Ukr. Acad. Sci., ICMP-91-56P, Lviv, 1991. Stasyuk I.V., Shvaika A.M., Schachinger E., Phys. C., 1993, 213, No. 1, 57.

8. Brandt U., Mielsch C., Z. Phys. B, 1989, 75, 365; 1990, 79, 295; 1991, 82, 37.

9. Stasyuk I.V., Cond. Matt. Phys., 2000, 3, 437.

10. Tserkovnikov Yu.A., Teor.i Mat. Fiz. 1971. No. 7, 260.

11. Plakida N.M., Phys. Lett. A, 1973, 43, 467.

12. Plakida N.M. Method of Two-Time Green's Functions in the Theory of Anharmonic Crystals. - In: Statistical physics and quantum field theory, ed. by N.N. Bogolyubov, Moscow, 1973, 240.

13. Hirsch J.E., Tang S., Phys. Rev. B, 1988, 40, No. 4, 2179.

\section{Спектр псевдоспін-електронної моделі у випадку наближення сплаву}

\author{
І.В.Стасюк, В.О.Краснов \\ Інститут фізики конденсованих систем, НАН України, \\ 79011 Львів, вул. Свєнціцького, 1
}

Отримано 26 січня 2006 р.

Досліджено спектр псевдоспін-електронної моделі у наближенні сплаву при використанні методу динамічного середнього поля (ДСП). Досліджується випадок двох близьких зон хаббардівського типу, що визначають положення хімічного потенціалу при заданих параметрах моделі. Розглянуто умови появи щілини в спектрі. Досліджено вплив поля асиметрії $h$ та тунельного розщеплення рівнів на критичне значення вузлової хаббардівської константи взаємодії $U_{\text {crit }}$.

Ключові слова: метод динамічного середнього поля, незвідні функції Гріна, псевдоспін-електронна модель, наближення сплаву

PACS: $71.10 . F d, 71.38 .+i$ 
